Brit. J. Ophthal. (1957) 41, 558.

\title{
A NEW MEDIUM FOR THE STORAGE OF DONOR EYES FOR CORNEAL GRAFTS*
}

\author{
BY
}

\section{A. SACHS}

From the Corneo-Plastic Unit and Regional Eye Bank, Queen Victoria Hospital, East Grinstead

THE EXPANSION of modern keratoplasty has increased the demand for suitable donor eyes, but for obvious reasons supplies are limited. This shortage has stimulated research into the best methods of preserving and storing donor eyes in order to eliminate any preventable factors which may be responsible for loss or wastage of this precious material.

Although it is not a universal practice, in this Corneo-Plastic Unit, no donor eye is used unless contamination by pathogenic bacteria has been absolutely excluded. During the period January, 1955, to June, 1956, 51 (41.3 per cent.) out of 138 post-mortem eyes, donated to this Bank for corneal grafting and stored in sterile liquid paraffin, were found on culture to be contaminated and unsuitable for use. The organisms isolated belonged to the staphylococcal, coliform, proteus, and corynebacterium groups. Contamination with a penicillin-sensitive strain of Staphylococcus albus is not regarded as a bar to the use of an eye for corneal grafting.

The high incidence of wastage through bacterial contamination in addition to the general shortage caused concern and prompted an investigation to find a more suitable storage medium than sterile liquid paraffin. The use of antibiotics was explored, but it was found that immersion of an enucleated eye for a short period in an antibiotic solution would not necessarily guarantee sterility. Moreover, on account of the high proportion of resistant bacteria, the use of penicillin was abandoned as unsafe. A combination of chloramphenicol and streptomycin has been found to give the most consistently satisfactory results when used in concentrations of $1,000 \mu \mathrm{g}$. per ml., but even then it is essential for the eye to be in contact with the antibiotics for at least 48 hours. This is presumably due to the time required for the antibiotics to penetrate to the deeper structures of the eye.

The criteria for the physiological requirements of corneal storage described by Davson (1955) formed the basis of the investigation which has resulted in the development of a new medium. After being stored for 14 days in 
this medium, eyes were histologically in a better condition than those stored in liquid paraffin, and the structures of the eye showed less degenerative changes. Corneae taken from eyes stored in the new medium have now been used in 26 cases, and the clinical results have been satisfactory. Also at operation it was found that the eyes were more easily handled and washed than those stored in liquid paraffin. Further, a possible source of failure of grafting due to contamination of the wound by liquid paraffin has been eliminated. For these reasons it has been decided that sterile liquid paraffin will be replaced by the new medium in this Eye Bank.

\section{Procedure}

The following procedure is now routine. Containers filled with the new medium are either issued by the laboratory to the Eye Bank or despatched direct to hospitals in small insulated ice-boxes for the use of colleagues who procure donor eyes in different parts of the country. The stocks of medium in hospitals are maintained and replaced as required.

Donor eyes are removed either by the medical staff of this Unit when they collect, or by colleagues who obtain the material on their behalf. As soon as possible after removal the eye is placed in the medium, and then despatched in the small ice-box to the Eye Bank. The eye is thus subjected to the sterilizing action of the medium during transit. On arrival at the Eye Bank it is stored at refrigerator or deep-freeze temperature until required for use. If there is any change in colour of the indicator from red to orange or yellow, or if the medium appears suspiciously turbid, the eye is immediately removed, cultured, and transferred to a fresh container of medium.

Further investigation is required to determine by tissue culture and other methods whether this new medium increases the longevity of the cornea beyond the accepted 10-day period when stored in other types of medium.

\section{Apparatus and Material required for Preparing the New Medium}

(1) The standard blood-transfusion bottle graduated in 100-ml. quantities, and fitted with a screw-cap and rubber liner, is the most suitable and convenient type of container for preparing bulk quantities of the medium.

(2) For the storage of eyes, wide-mouth specimen jars of 2-oz. fitted with an aluminium screw-cap and wax liner are used (these are issued by the Supply Branch of the Ministry of Health).

(3) A rubber bung to fit the blood transfusion bottle is fitted with an air inlet containing a cotton-wool filter, and a piece of glass tubing bent at an angle of $90^{\circ}$. The bung is sterilized separately and fitted to the bottle before the jars are filled.

\section{Ingredients of Medium}

(a) Intradex (6 per cent. dextran in normal saline) (Glaxo)

(b) Dextrose 5 per cent. sterile solution in distilled water 
(c) Phenol red sterile solution 1:1,000

(d) Chloramphenicol

(e) Streptomycin

(f) Standard nutrient agar (issued by the Southern Group Laboratory). This is melted in a steamer before use.

It is essential that the chemical constituents of any medium should be as nearly as possible in equilibrium with those of the aqueous humour and the tears which bathe the posterior and anterior surfaces of the cornea for the purpose of viability and nourishment and to maintain the hydration of the cornea. It is also essential to prevent any osmotic alteration in the volume of the globe. In practice, however, it has been found that a slightly hypertonic solution is preferable, as this prevents the cornea from imbibing fluid and becoming oedematous. The eye does lose some volume, but it is considered that the slight hypertonicity is justified in that it prevents excessive hydration of the cornea which favours disintegration of the supporting protein scaffold of the cells and autolysis of the protein.

Dextran in solution is selected on account of the size of its molecules and its properties as a plasma expander to maintain equilibrium with the intra-ocular proteins of the aqueous humour and the vitreous body.

The isotonic solution of dextrose is used both to maintain equilibrium with the diffusible ions and molecules in the aqueous and vitreous body and to provide nourishment for the cornea.

The phenol red indicator is added to enable the $\mathrm{pH}$ to be checked and to detect contamination. When bacteria are present, the indicator changes from red $\rightarrow$ orange $\rightarrow$ yellow. It has been found that a change in $\mathrm{pH}$ does occur after 14 days' storage, but the medium has been proved to be sterile after culture; it is believed that this change in colour is indicative of metabolism of the dextrose by the eye during storage.

Nutrient agar (to a concentration of 0.32 per cent. agar) is used to increase the viscosity of the medium and to act as a vehicle and buffer to protect the cornea during transit and storage.

The $15 \mathrm{ml}$. nutrient agar added to each container is to economize in the amount of medium required and also to provide a cushion for the eye at the bottom of the container.

\section{Preparation of Medium}

The medium is prepared in 500-ml. quantities (this being sufficient to fill fourteen or fifteen jars). The ingredients are added to a sterile bottle in the following order:

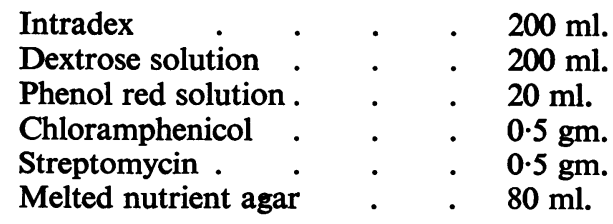

The bottle is well shaken to ensure thorough mixing of the contents; it is sterilized at $20 \mathrm{lb}$. pressure for 30 minutes and then cooled to $60^{\circ} \mathrm{C}$. before the jars are filled. After sterilization the $\mathrm{pH}$ is $7 \cdot 4$ to $7 \cdot 6$. Owing to its viscosity it is essential that the medium be kept at a temperature of $60^{\circ} \mathrm{C}$. when filling the jars. 


\section{Summary}

(1) A new medium for the storage of post-mortem donor eyes for corneal grafting is described.

(2) The high rate of potential infection (41 per cent.) of post-mortem donor eyes has been greatly diminished and the wastage of eyes has been reduced.

(3) The method has been proved to be valuable in practice. The medium is simple to prepare, avoids oil contamination of the graft, and ensures sterility which can be seen at a glance. Delay for culture report is thus eliminated.

(4) The medium also helps to nourish the graft during storage.

(5) Further research is required to determine whether storage in the new medium increases the optimum period during which an eye may be stored.

I wish to acknowledge the help and encouragement I have received from Mr. B. W. Rycroft and his assistants Mr. G. Romanes and Dr. M. Garber during the investigations.

\section{REFERENCE}

Davson, H. (1955). In “Corneal Grafts", ed. B. W. Rycroft, chap. 2, p. 34. Butterworth, London. 